\title{
Less Intensive 1 (LI1) Trial: A Randomized Phase II/III Clinical Trial in Approximately 1000 Elderly Patients With Acute Myeloid Leukaemia (AML) Deemed Not Suitable for Intensive Chemotherapy
}

Ian Thomas ( $\nabla$ thomasif@cardiff.ac.uk)

Cardiff University Centre for Trials Research https://orcid.org/0000-0002-8395-8075

Mike Dennis

The Christie NHS Foundation Trust

Laura Upton

Cardiff University Centre for Trials Research

Cono Ariti

Cardiff University Centre for Trials Research

Nigel Russell

Nottingham University

Robert Hills

University of Oxford

Mhairi Copland

University of Glasgow

Alan Burnett

Blackwaterfoot

\section{Research Article}

Keywords: AML, leukaemia, elderly, non-intensive, chemotherapy, randomized, Pick a Winner, cytarabine, international, cancer

Posted Date: June 24th, 2021

DOl: https://doi.org/10.21203/rs.3.rs-434566/v1

License: (1) This work is licensed under a Creative Commons Attribution 4.0 International License.

Read Full License 


\section{Title}

The Less intensive 1 (LI1) trial is a randomized phase II/III clinical trial in approximately 1000 elderly patients with acute myeloid leukaemia (AML) deemed not suitable for intensive chemotherapy. The trial compares novel agents against the standard treatment (low dose cytarabine, LD Ara-C), using a Pick-a-Winner design.

\section{Names protocol contributors}

Chief Investigator: Dr Mike Dennis (2016 - present)

Co-Cl: Prof Mhairi Copland

Clinical Coordinators: Prof Alan Burnett (Chief Investigator 2011-2015), Prof Nigel Russell, Prof

Richard Clark

Trial Design and statistical oversight: Prof Robert Hills

Statistical analysis: Mr Cono Ariti

\section{Abstract}

- Background: Acute myeloid leukaemia (AML) is a heterogeneous disease where outcome is substantially influenced by age. Over the last 30-40 years, significant improvements have been made in survival for younger patients (those under 60). However, with the median age of the disease at diagnosis at 65 years, outcome in older patients has improved much less. Additionally, many elderly patients are not considered suitable for intensive chemotherapy and the current standard treatment is considered unsatisfactory.

- Methods: LI1 will evaluate several relevant therapeutic questions in AML patients over 60 years of age for whom conventional chemotherapy is not considered suitable.

Patients are invited to enter a randomized comparison of standard therapy (LD Ara-C) versus a novel treatment. A number of different treatment options are available at any one time, but there is no comparison between novel agents. If, at initial evaluation, a novel agent is deemed unlikely to demonstrate the required improvement, it may be removed from the protocol and replaced with another novel agent, via protocol amendment. Agents are initially entered into a phase II comparison against standard of care - where an agent is considered promising at interim review (based on remission rates), the randomisation may be extended to a fully powered phase III comparison based on survival.

Agents which may prolong survival or improve remission rates will also be required to demonstrate equivalence or better of quality of life in patients - therefore, quality of life assessments are undertaken throughout, in each arm.

- Discussion: Even when less intensive treatment options are delivered, the outcomes are not satisfactory. The National Cancer Research Institute (NCRI) AML working group has a strong network, required for delivery of this trial program. The UK network is augmented by international collaborative groups, most notably from New Zealand and Denmark. The use of the Pick-a-Winner design improves efficiency and speed of review of the available novel agents with the aim of benefitting the increasing number of patients in this age group.

- Trial registration: ISCRTN40571019, EUDRACT2011-000479-19 (12 ${ }^{\text {th }}$ May 2011)

\section{Keywords}

AML, leukaemia, elderly, non-intensive, chemotherapy, randomized, Pick a Winner, cytarabine, international, cancer

\section{Administrative information}




\begin{tabular}{|c|c|}
\hline Title $\{1\}$ & $\begin{array}{l}\text { The Less intensive } 1 \text { (LI1) trial is a randomized phase II/III } \\
\text { clinical trial in approximately } 1000 \text { elderly patients with acute } \\
\text { myeloid leukaemia (AML) deemed not suitable for intensive } \\
\text { chemotherapy. The trial compares novel agents against the } \\
\text { standard treatment (low dose cytarabine, LD Ara-C), using a } \\
\text { Pick-a-Winner design }{ }^{1} \text {. }\end{array}$ \\
\hline Trial registration $\{2 \mathrm{a}$ and $2 \mathrm{~b}\}$. & $\begin{array}{l}\text { The trial is registered on ISRCTN } 40571019 \text { and EUDRACT 2011- } \\
000749-19\end{array}$ \\
\hline Protocol version $\{3\}$ & $\begin{array}{l}\text { This protocol paper describes a number of different protocol } \\
\text { versions between v1.4 (June 2011) and v11.1 (October 2019) }\end{array}$ \\
\hline Funding $\{4\}$ & $\begin{array}{l}\text { The trial was supported by central funding from Blood Cancer } \\
\text { UK (formerly Bloodwise, formerly Leukaemia Lymphoma } \\
\text { Research), and received additional funding from the } \\
\text { collaborating pharma companies (Cyclacel, Daiichi Sankyo } \\
\text { (formerly Ambit Biosciences), Sunesis Pharmaceuticals, Synta } \\
\text { Pharma, Karyopharm, Cell Therapeutics Inc, Celgene and } \\
\text { BioCancer Therapeutics Inc) }\end{array}$ \\
\hline Author details $\{5 a\}$ & $\begin{array}{l}\text { Senior Research Fellow: } \\
\text { Ian Thomas, Centre for Trials Research, Cardiff University } \\
\text { Chief Investigator (CI): } \\
\text { Dr Mike Dennis, Consultant Haematologist, The Christie } \\
\text { Hospital, Manchester, UK } \\
\text { Co-Cl: } \\
\text { Prof Mhairi Copland Professor of Translational Haematology } \\
\text { and Honorary Consultant, Director of the Paul O'Gorman } \\
\text { Leukaemia Research Centre and Head of Experimental } \\
\text { Haematology, College of Medical, Veterinary and Life Sciences, } \\
\text { Institute of Cancer Sciences, University of Glasgow } \\
\text { Clinical Coordinators: } \\
\text { Prof Alan Burnett, Blackwaterfoot, Isle of Arran, UK (previous } \\
\text { CI) } \\
\text { Prof Nigel Russell, Consultant Haematologist, Guy's Hospital } \\
\text { NHS Trust (Emeritus Professor of Haematology, } \\
\text { Nottingham University) } \\
\text { Trial Design, statistical oversight and statistical analysis: } \\
\text { Prof Robert Hills, Professor of Medical Statistics, Nuffield } \\
\text { Department of Population Health, University of Oxford } \\
\text { Statistical analysis: } \\
\text { Mr Cono Ariti, Research Fellow (Statistics), Centre for Trials } \\
\text { Research, Cardiff University }\end{array}$ \\
\hline
\end{tabular}




\begin{tabular}{|l|l|}
\hline $\begin{array}{l}\text { Name and contact information } \\
\text { for the trial sponsor }\{5 \mathrm{~b}\}\end{array}$ & $\begin{array}{l}\text { Chris Shaw, Research Governance Coordinator, Research } \\
\text { Integrity, Governance and Ethics Team, Research and } \\
\text { Innovation Services, Cardiff University, 7th Floor, Mackenzie } \\
\text { House, 30-36 Newport Road, Cardiff, CF24 ODE, 02920 879130, } \\
\text { shawc3@cardiff.ac.uk }\end{array}$ \\
\hline Role of sponsor $\{5 \mathrm{c}\}$ & $\begin{array}{l}\text { The trial Sponsor delegated all duties regarding the } \\
\text { management of the trial to the Haematology Clinical Trials Unit } \\
\text { (HCTU), which became part of the Centre for Trials Research, } \\
\text { School of Medicine, Cardiff University during the trial. Sponsor } \\
\text { has been a signatory on all contracts with collaborating partners } \\
\text { (external collaborators, pharma companies, etc.). Sponsor has } \\
\text { maintained representation on the Trial Management Group } \\
\text { throughout the trial. The TMG has authorised and reviewed this } \\
\text { report. }\end{array}$ \\
\hline
\end{tabular}

\section{Introduction}

\section{Background and rationale $\{6 a\}$}

A substantial majority of patients diagnosed with AML or high risk myelodysplastic syndrome (MDS) are elderly and either decline, or are not considered fit for, intensive treatment.

As part of the NCRI/LRF AML14 trial in the late 1990/early 2000s², low dose Ara-C (LD Ara-C) was compared with hydroxycarbamide. The trial was closed early because LD Ara-C was significantly superior. Although an $18 \%$ remission rate was observed, the overall survival (OS) was still poor at a median of 5 months. Benefit was limited to patients who achieved remission, which therefore became a major objective against which to assess new treatments. In the AML14 trial no patients who had high-risk cytogenetics responded to LD Ara-C, however in the cohort of patients who were allocated to LD Ara-C in the follow-up NCRI trial (AML16) the marrow remission rate was $11 \%$. The survival of patients in this patient group was deemed unsatisfactory and, the time of trial design and outset, no treatment had shown statistical superiority to the LD Ara-C schedule outlined in this protocol for this patient group.

The aim of the LI-1 trial is to identify treatments which are superior to LD Ara-C 20mg given bd as part of a 10-day course - as such, the aims of the LI1 trial could be regarded as a broad continuation of the non-intensive arm of the AML16 trial. The protocol has already, and further aims, to evaluate a number of different therapies compared to LD Ara-C in a randomised fashion. Enhanced pharmacovigilance is used to monitor the ongoing safety of the patients; there is also the built-in option to reject, at an early stage, treatments which are unlikely to provide sufficient survival improvement to become a new standard of care, thus protecting patients from continued exposure to agents which are unlikely to be of benefit. Comparison has been and will be contemporaneous, between novel agents and controls randomised to LD Ara-C - the simultaneous randomisation between different novel options provides economy and allows the questions to be answered with scientific rigour with fewer patients than successive trials of single novel arms vs control. The novel options described here were, and are, available only to patients within the LI1 trial. The overall aim is to identify promising agents that can be carried forward to a phase III comparison with standard of care.

\section{Objectives $\{7\}$}

The primary objective of the protocol is to compare LD Ara-C versus a number of available novel approaches that were available at trial onset and additional agents that have been added to the 
trial protocol via substantial amendment.

Blood and bone marrow have been provided by participating trial centres at diagnosis and, when available, at relapse to allow evaluation of the therapeutic relevance of morphological, cytogenetic, molecular-genetic and immunophenotypic assessments. The relevance of molecular characteristics and cytogenetic abnormalities and other biomarkers to response to treatment are investigated and reported per randomisation arm.

An important additional aim is to store diagnostic tissue for future research under the custodianship of the Sponsor, Cardiff University.

\section{Trial design $\{8\}$}

AMLLI-1 is part of an ongoing programme of development of treatment primarily for older patients with AML and high-risk MDS who are not considered suitable for intensive chemotherapy. It should be noted that this trial protocol was a continuation of the non-intensive arm of the AML16 trial (EUDRACT 2005-002847-14) and the authors note the considerable input of the Birmingham Clinical Trials Unit in the initial trial design - in particular, Prof Keith Wheatley and Elizabeth Sellwood.

AML LI-1 is a controlled multi-centre, multi-arm, contemporaneously randomised, seamless Phase II/III parallel-group trial utilising a 'Pick-a-winner'3 ${ }^{3}$ design. Treatment allocation is a 1:1 ratio. Patients are invited to enter a comparison of standard therapy (LD Ara-C) versus a novel treatment. In the event of any of the novel treatments appearing superior on preliminary analyses, the comparison continues in a Phase III design.

With LD Ara-C, nearly all patients in the non-intensive arm of the AML16 trial died within 3 years. It was unrealistic to expect any of the treatments being evaluated in this Programme to lead to absolute improvements in two-year survival of more than approximately $10 \%$ to $15 \%$ - however, benefits which are too small would probably not be worthwhile given the likely costs of the new agents under investigation. Therefore, the objective of the trial group has been to double 2-year survival from the historical rate of approximately $11 \%$ seen in AML14, to around $22 \%^{2}$. Due to the heterogeneity of the disease, interpretation of single-arm studies is fraught with difficulties arising from different patient populations, and there is a requirement to demonstrate that a new treatment provides an improvement in care compared to standard of care LD Ara-C. Randomised evaluation of the novel agents is a pre-requisite in this research environment. Participating centres are normally expected to randomise patients between all the available options at their centres. For example, where 2 novel treatments are compared against LD Ara-C, the randomisation allocation is 1:1:1. If a patient is not eligible for one of the treatments (either due to operational restrictions or due to the patient not meeting the eligibility criteria for a specific arm), they will be randomised between the options for which they are eligible.

\section{Methods: Participants, interventions and outcomes Study setting $\{9\}$}

The participants in this trial are patients with AML and are referred to accordingly throughout. Patients will often present with initial symptoms to their general practitioner (GP) and be referred to a haemato-oncological centre for further examination. Diagnosis of AML or MDS is based on a bone marrow result under the clinical team at the treating, usually regional, centre.

The trial is supported by the NCRI and as such is available to all suitable treatment centres in the UK. Information about the participating centres is made publicly available at:

https://www.cancerresearchuk.org/about-cancer/find-a-clinical-trial/a-trial-looking-sapacitabineAC220-vosaroxin-cytarabine-acute-myeloid-leukaemia-aml-li-1

The trial has also been supported by collaborative groups in Denmark, New Zealand, France and Australia, where treatment centres offer novel agents in compliance with ongoing and current national regulatory approvals. 


\section{Eligibility criteria $\{10\}$}

Patients who have provided written informed consent have been eligible for the LI-1 trial if:

- They have one of the forms of acute myeloid leukaemia, except Acute Promyelocytic Leukaemia as defined by the World Health Organisation (WHO) Classification this can be any type of de novo or secondary AML - or high risk Myelodysplastic Syndrome, defined as greater than $10 \%$ marrow blasts (RAEB-2).

- They are over the age of 60

- They are considered by the investigator to be unfit for intensive chemotherapy Introduction of the novel agents is often accompanied by additional exclusion criteria specific to those agents - for instance, patients with pre-existing cardiac or liver toxicities may be excluded from allocation to some of the novel treatments.

Patients are (and continue to be) excluded from the trial if:

- They are less than 60 years of age

- They have previously received cytotoxic chemotherapy for AML. [Hydroxycarbamide, or similar low-dose therapy, to control the white count is not an exclusion criterion].

NB: Previous treatment with a demethylating agent for low grade MDS ( $<10 \%$ blasts) was not an exclusion.

- They are in blast transformation of Chronic Myeloid Leukaemia (CML)

- They have a concurrent active malignancy under treatment.

- They are pregnant or lactating.

- They have Acute Promyelocytic Leukaemia.

- Known infection with human immunodeficiency virus (HIV)

- Total bilirubin $\geq 1.5$ x ULN, unless Gilbert's syndrome

- Aspartate aminotransferase (AST) / Alanine aminotransferase (ALT) $\geq 2.5 \times$ ULN and/or alkaline phosphatase $\geq 2.5 \times$ ULN

- Serum creatinine $\geq 175 \mu \mathrm{mol} / \mathrm{L}$

- History of myocardial infarction (MI), unstable angina, cerebrovascular accident, or transient ischemic attack (CVA/TIA) within 6 months

\section{Who will take informed consent? $\{26 a\}$}

Consent forms are provided by the coordinating trial team and localised with institutional details. Trial consent is gathered by the clinical team (investigator or nurse) at the centres participating in the trial. Copies of the consent forms are held in the patient's notes, and confirmation of the patient's informed consent is required in order for the patient to be randomised into the trial.

\section{Additional consent provisions for collection and use of participant data and biological specimens $\{26 \mathrm{~b}\}$}

Additional consent has also been requested specifically for ongoing storage of the patients' samples for future research, under the auspices of the Sponsor, Cardiff University. Patients are free to enter the trial, and be allocated any of the available treatment arms, without consenting for ongoing storage of their samples. Sharing of data is subject to existing Centre for Trials Research (CTR) procedures, consistent with ongoing regulations and the patient's consent. Formal CTR SOPs replaced existing HCTU SOPs when HCTU became part of the Centre for Trials Research in 2015. 
Novel agents are selected based on promising results in other disease areas or due to promising early-phase results. Justification for the inclusion of each novel therapy is subject to scientific review on behalf of the Sponsor, and ethical review on behalf of the Research Ethics Committee.

\section{Intervention description $\{11 a\}$}

\begin{tabular}{|c|c|c|c|}
\hline Standard treatment & Details & Opened & Current status \\
\hline LD Ara-C & $\begin{array}{l}\text { Ara-C } 20 \mathrm{mg} \text { bd by subcutaneous injection daily on days } 1-10 \text { ( } 20 \text { doses) to be repeated } \\
\text { at } 28 \text { to } 42 \text { day intervals }\end{array}$ & January 2012 & Open \\
\hline \multicolumn{4}{|c|}{ The following treatment arms were included in the trial design at outset in 2012} \\
\hline Novel treatment & Details & Opened & Current status \\
\hline Sapacitabine only & $\begin{array}{l}\text { 300mg orally b.i.d. for } 3 \text { consecutive days in week one and in week two (randomisation } \\
\text { was carried over from previous trial, AML16 and was available from trial opening). This } \\
\text { was followed by a minimum of } 4 \text { weeks of no treatment }\end{array}$ & January 2012 & $\begin{array}{l}\text { Randomisation } \\
\text { closed }\end{array}$ \\
\hline Vosaroxin only & $\begin{array}{l}72 \mathrm{mg} / \mathrm{m}^{2} \text { intravenously over } 10 \text { minutes on days } 1 \text { and } 4 \text { of each treatment course (two } \\
\text { doses), with treatment courses repeated every } 4-6 \text { weeks }\end{array}$ & February 2012 & $\begin{array}{l}\text { Randomisation } \\
\text { closed }\end{array}$ \\
\hline LD Ara-C + vosaroxin & $\begin{array}{l}\text { LD Ara-C and vosaroxin were administered as above, with both treatments starting on } \\
\text { the same day (day } 1 \text { of the treatment course), repeated every } 4-6 \text { weeks }\end{array}$ & June 2012 & $\begin{array}{l}\text { Randomisation } \\
\text { closed }\end{array}$ \\
\hline LD Ara-C + AC220 & $\begin{array}{l}\text { LD Ara-C was adminstered as above. The dose of AC220 was } 60 \mathrm{mg} \text { ( } 2 \times 30 \mathrm{mg} \text { tablets) } \\
\text { orally, daily for } 21 \text { consecutive days commencing on day } 1 \text { of each course of LD Ara-C } \\
\text { treatment, irrespective of when each course of LD Ara-C started }\end{array}$ & July 2012 & $\begin{array}{l}\text { Randomisation } \\
\text { closed }\end{array}$ \\
\hline \multicolumn{4}{|c|}{ The following treatment arms were added to the trial protocol via protocol amendment } \\
\hline Novel treatment & Details & Opened & Current status \\
\hline $\begin{array}{l}\text { LD Ara-C + } \\
\text { ganetespib }\end{array}$ & $\begin{array}{l}\text { LD Ara-C was administered as above. } 120 \mathrm{mg} / \mathrm{m}^{2} \text { ganetespib was given as a } 1 \text { hour } \\
\text { intravenous infusion commencing on day } 1 \text { of each LD Ara-C treatment course. This } \\
\text { was repeated on days } 8,15,22 \text { and } 29 \text { of each LD Ara-C course }\end{array}$ & November 2012 & $\begin{array}{l}\text { Randomisation } \\
\text { closed }\end{array}$ \\
\hline $\begin{array}{l}\text { LD Ara-C + } \\
\text { tosedostat }\end{array}$ & $\begin{array}{l}\text { LD Ara-C was administered as above. Tosedostat was given at a dose of } 180 \mathrm{mg} \text { ( } 3 \\
\text { capsules) orally once a day. Treatment commenced on day } 1 \text { of the first course of LD } \\
\text { Ara-C and continued daily for } 6 \text { months }\end{array}$ & June 2014 & $\begin{array}{l}\text { Randomisation } \\
\text { closed }\end{array}$ \\
\hline LD Ara-C + selinexor & $\begin{array}{l}\text { LD Ara-C was administered as above. For the first two cycles of LD Ara-C, selinexor was } \\
\text { administered on days } 1 \& 3 \text { of each week between LD Ara-C courses. The dose of } \\
\text { Selinexor was } 30 \mathrm{mg} / \mathrm{m} 2 \text {, starting at day } 12 \text {, where day } 1 \text { was the first day of LD Ara-C. }\end{array}$ & June 2014 & $\begin{array}{l}\text { Randomisation } \\
\text { closed }\end{array}$ \\
\hline $\begin{array}{l}\text { LD Ara-C + } \\
\text { lenalidomide }\end{array}$ & $\begin{array}{l}\text { LD Ara-C administered as above. Lenalidomide was administered orally once daily in a } \\
\text { flat } 10 \mathrm{mg} \text { dose for } 21 \text { days, where day } 1 \text { was day } 1 \text { of LD Ara-C. This course was } \\
\text { repeated after a } 2 \text {-week rest period and continued for four courses (therefore } \\
\text { repeated every } 5 \text { weeks) }\end{array}$ & January 2017 & $\begin{array}{l}\text { Randomisation } \\
\text { closed }\end{array}$ \\
\hline LD Ara-C + BCT100 & $\begin{array}{l}\text { LD Ara-C administered as above. BCT- } 100 \text { dose is } 1600 \mathrm{U} / \mathrm{kg} \text { on Days } 1,8,15 \text { and } 22 \text { as a } \\
1 \text { hour IV infusion. This regimen is repeated for up to a total of } 4 \text { cycles of treatment }\end{array}$ & September 2018 & Open \\
\hline
\end{tabular}

\section{Criteria for discontinuing or modifying allocated interventions $\{11 \mathrm{~b}\}$}

The aim for all treatments throughout the trial was to administer 4 courses. If the patient was benefitting after 4 courses, treatment could continue until disease progression.

AC220 dosing could be interrupted for up to 14 days if the absolute central laboratory value of the QTcF interval increases to $>500 \mathrm{~ms}$. Where patients receiving AC220 experienced a Grade 3 or 4 non-haematological toxicity at the initial $90 \mathrm{mg}$ dose, the dose could be reduced to $60 \mathrm{mg}$ in certain circumstances.

Patients allocated to receive sapacitabine would receive treatment on Day 8 of Cycle 1 and on Day 1 and 8 of all subsequent cycles when clinically significant and drug-related non-hematologic toxicities had resolved to grade 1 or baseline. After recovery, a dose reduction of $50 \mathrm{mg}$ b.i.d. could be instituted for grade 3-4 drug-related non-hematologic toxicities for the next treatment 
cycle or next week of dosing. For patients who experienced a $>25 \%$ decrease in bone marrow blasts, a dose reduction of $50 \mathrm{mg}$ b.i.d. for delay in recovering absolute neutrophils and platelets to baseline or best level on trial beyond day 42 was instigated.

Patients receiving vosaroxin who experienced clinically significant National Cancer Institute (NCl) Common Terminology Criteria for Adverse Events, v3.0 (CTCAE) Grade 3 or 4 received a reduced dose of vosaroxin according to rules described in the table below

\begin{tabular}{|c|c|}
\hline Occurrence of Grade $\mathbf{3}$ or $\mathbf{4}$ Non-haematologic Toxicity & Action \\
\hline First Incident & $\begin{array}{c}\text { Reduce vosaroxin injection dose from } 72 \\
\mathrm{mg} / \mathrm{m} 2 \text { to } 60 \mathrm{mg} / \mathrm{m} 2\end{array}$ \\
\hline Second Incident & $\begin{array}{c}\text { Reduce vosaroxin injection dose from } 60 \\
\mathrm{mg} / \mathrm{m} 2 \text { to } 50 \mathrm{mg} / \mathrm{m} 2\end{array}$ \\
\hline Third Incident & Discontinue patient from trial treatment \\
\hline
\end{tabular}

Patients receiving Selinexor who experienced associated fatigue (not reduced to an acceptable level with supportive care measures), received a dose reduction of $30 \%$. Once-weekly dosing (at the higher dose of $40 \mathrm{mg}$ ) could also be considered in patients whose disease was highly responsive to Selinexor.

For patients receiving ganetespib, diarrhoea was the primary concern. If diarrhoea reached grade 3 or 4 , subsequent courses of ganetespib were given at $80 \%$ dose level. If grade 3 or 4 diarrhoea recurred ganetespib treatment was discontinued. For liver toxicity, if AST or ALT reached $>3 x$ but $<$ $5 x$ ULN and total bilirubin was $<2 x$ ULN and recovery had taken place, subsequent doses were reduced to $80 \%$. For renal function, if serum creatinine rises to $>3 x$ ULN dosing was delayed until recovery.

Dose reduction (to $120 \mathrm{mg} /$ day) or interruption were considered an appropriate way for investigators to manage an adverse event $(A E)$ considered to be related to tosedostat administration or an $\mathrm{AE}$ where continued tosedostat administration could impair recovery. Interruptions/reductions of up to 2 weeks were considered acceptable.

If patients who were receiving lenalidomide developed a grade 3 or 4 non-haematological toxicity, lenalidomide dose reduction to $5 \mathrm{mg}$ per day was permitted. If well-tolerated for 1 course, consideration was given to reintroduce the $10 \mathrm{mg}$ dose for the following course.

As above, the BCT100 randomisation remains open. If patients receiving $\mathrm{BCT} 100$ develop a grade 3 or 4 non-haematological toxicity, BCT-100 dose reduction to 1200 Units $/ \mathrm{kg}$ ( $25 \%$ dose reduction) is considered appropriate and continued for further cycles in combination with LD Ara-C.

\section{Strategies to improve adherence to interventions $\{11 \mathrm{c}\}$}

The adherence of patients to their allocated treatment has not been monitored centrally in this phase II/III trial. However, data collection at participating centres includes confirmation as to whether patients received the full allocated protocol treatment. Where patients did not receive allocated treatment (usually a dose reduction, or missed doses due to toxicity), details of the treatment given have been collected.

\section{Relevant concomitant care permitted or prohibited during the trial $\{11 \mathrm{~d}\}$}

Patients were not permitted to be allocated to AC220 or ganetespib treatment if they were receiving any of the potentially QTc-prolonging medications listed in the trial protocol. Exceptions were made for antibiotics and antifungals that were used as standard of care for the prevention or treatment of infections or if the treating clinician believed that beginning therapy 
with a potentially QTc-prolonging medication was vital to an individual patient's care while on trial. The decision as to whether such a medication was used was taken in the context of the patient's medical history and current QTc values.

\section{Provisions for post-trial care $\{30\}$}

Potential legal liability of harm to the patients in this trial arising from the management and design of the research was covered by the Sponsor's insurance/indemnity arrangements. Legal liability for the conduct of the research was covered by the NHS and professional indemnity arrangements of the participating centres. Ongoing supply of novel agents to patients who were deemed to be benefitting from treatment was arranged between the pharmaceutical collaborators and the Sponsor and made available to patients for as long as supply was available to the Sponsor.

\section{Outcomes $\{12\}$}

All outcomes are as defined by IWG criteria as described in Cheson BD et al ${ }^{4}$. Revised recommendations of the International Working Group for Diagnosis, Standardization of Response Criteria, Treatment Outcomes, and Reporting Standards for Therapeutic Trials in Acute Myeloid Leukemia. J Clin Oncol. 2003 Dec 15; 21(24):4642-9.

\section{Primary outcomes}

- Overall survival - time from randomisation to death from any cause. Survival will be used to examine the comparison of LD Ara-C with the novel therapy. Patients will be censored at the point of withdrawal of consent or last known follow-up.

- Complete remission ( $C R+C R i)$ achievement. Remission rates will be used to examine the comparison of LD Ara-C with the novel therapy.

- Duration of response (CR, CRi), relapse rates and deaths in first CR. Relapse-free survival (RFS) will be used to examine the comparison of LD Ara-C with AC220. RFS will censor patients at the point of withdrawal of consent or last known follow-up.

Definition of Complete Marrow Remission (CR):

- Cellularity of marrow should be at least $20 \%$ with evidence of tri-lineage regeneration.

- Less than $5 \%$ blasts.

- No Auer rods.

- No extra-medullary disease.

- Haematological recovery times: from the end of the course to the recovery of Peripheral blood count (platelets) recovery to $100 \times 10^{9} / \mathrm{I}$ or Neutrophil recovery to $1.0 \times 10^{9} / \mathrm{I}$, censored at the next course or time last known to have been recovered

Definition of CR with incomplete recovery (CRi)

As for CR but without recovery either or both platelets or neutrophils to the levels required for CR. Patient required to be platelet-transfusion independent.

Secondary outcomes

- Toxicity, both haematological and non-haematological.

- Supportive care requirements. This will include days in hospital, blood product usage, and days on antibiotics.

- Quality of Life Assessment. This will be undertaken at baseline (prior to start of treatment), 3 months, 6 months and 12 months. The instruments used will be the QLQc-30 including HAD score which has been validated in acute leukaemia, and the EuroQoL EQ-5D ${ }^{5}$. Patients should be able to undertake the assessment themselves.

\section{Participant timeline $\{13\}$}

Patients with AML in this trial receive a course of treatment every 4-6 weeks. Progression to the 
next course of treatment is often assessed on the status of the patient's blood and bone marrow, and accompanying toxicities (such as any renal or hepatic impairment). Subsequent treatment courses in this population are often delayed and, while the protocol describes 4 courses of treatment, many patients received 2-3 courses before disease progression.

Depending on the novel agent allocated, the treatment schedule was usually administered alongside the standard (LD Ara-C) schedule, starting on the same day. The treatment schedules for the novel agents are outlined in Intervention Description above.

Where the novel agent was an oral compound, patients usually self-administered in order to be discharged during treatment. LD Ara-C may be given as a self-administered subcutaneous injection, minimising the support required during the treatment course, and this was authorised throughout the trial.

\section{Sample size $\{14\}$}

The large improvements in survival of younger patients with AML observed over the last 40 years have, unfortunately, not been mirrored in older patients - in the intensive arm of AML14, survival at 5 years in patients aged 60 or over is only $15 \%$, while even with low-dose Ara-C nearly all patients in the non-intensive arm have died within 3 years. Thus, it is unrealistic to expect any of the treatments being evaluated in the Programme to lead to improvements in survival of more than $10 \%$ to $15 \%$, while smaller benefits would probably not be worthwhile given the likely costs of the new agents under investigation.

Experience within AML16 indicates that approximately 180 patients per annum can be recruited to the Programme. Initially, the protocol described the process as such:

To detect a 10\% absolute difference in 2 year survival from 10\% with LD AraC to $20 \%$ with a novel therapy (at a 2-tailed p-value of 0.01 with $80 \%$ power) would require about 200 patients and 170 deaths per arm. Thus, for novel therapies that are taken forward for fullscale Phase III evaluation, the aim will be to accrue 200 patients to each arm. Not all patients will be submitted for randomisation between all available arms, and not all arms will be available to all sites at any one time, but with up to 1000 patients in total over a 5 year accrual period there will be sufficient numbers to achieve the target of 200 per arm for promising treatments, allowing for the fact that not all treatments are likely to proceed to full evaluation.

This evaluation will take place in three stages. The precise approach for performing these initial assessments of effectiveness will depend on the anticipated modality of the treatment. For some treatments, it is anticipated that any improvements in survival will be achieved by increasing the number of durable remissions. For others, such as hypomethylating agents, it may be possible to improve survival without increasing the remission rate.

For treatments where the proposed effect is to improve survival by inducing a greater number of remissions, recruitment will proceed until at least 50 patients have entered each comparative arm (Ara-C and novel therapy). This component will then be analysed using CR as endpoint. While this assessment is taking place, recruitment may continue subject to the wishes of the DMEC. If the arm appears sufficiently promising, then recruitment will continue until 100 patients are in each arm. At this point, a similar analysis on CR will be undertaken. If, on the basis of examining the data from the first 100 patients in each arm, the novel treatment is sufficiently promising then recruitment will continue to 200 patients per arm as a Phase III study, when the trial endpoints will be changed to $C R$, relapse and overall survival. However, if at either of the earlier analysis points, the judgement is that the treatment is unlikely to hold promise, the comparison will be discontinued. To allow for the flexible trial design, where patients may enter either a full randomisation or any pairwise comparison with Ara- $C$, the trial will be analysed stratified by choice of comparison, using standard meta-analytic techniques. For the treatments in this protocol other than sapacitabine, the default stopping rule is as follows: assuming a CR rate of $15 \%$ with low dose Ara-C and aiming 
to identify new treatments that produce a CR rate of $30 \%$ or more, a minimum of 50 patients will be accrued to each novel therapy arm; if the improvement in CR rate in the novel therapy arm is less than $2.5 \%$ (15\% to 17.5\%), that arm will be closed (with a $7 \%$ chance of rejecting a treatment with a true $C R$ rate of $30 \%$ or more); if the difference in CR rates exceeds $2.5 \%$, the arm will continue to 100 patients. At this point, if the improvement in CR rate in the novel therapy arm is less than $7.5 \%$, that arm will be closed (with a further $8 \%$ chance of rejecting a treatment with a true $C R$ rate of at least 30\%); if the improvement in CR rate exceeds $7.5 \%$, the comparison will continue to the full 200 patient per arm trial. Under this scenario, the overall power to identify an effective new treatment will be about $85 \%$, and all but $7 \%$ of totally ineffective treatments will be dropped after either 50 or 100 patients.

While the benefit derived from some treatments is expected to come from an increase in the number of durable remissions, some drugs, notably hypomethylating agents give the promise that survival may be prolonged even without the achievement of a complete remission. In this case, an interim analysis based on complete remission is inappropriate. Instead, analyses will be based on overall survival once one quarter and one half of the required number of events have been seenin this case this equates to 85 and 170 events. At this point, the decision to stop or continue will be made on the basis of the hazard ratio for overall survival. A doubling of survival from $10 \%$ to $20 \%$ at two years equates to a hazard ratio of 0.70 . Thus, for drugs such as sapacitabine, where remission is not viewed as a prerequisite for survival, after 85 events, the hazard ratio (drug versus Ara-C) must not exceed 1; and after 170 events it must not exceed 0.85 (i.e. half the required effect). Simulations show similar power under these requirements to the scenario for $C R$ given above.

It is important to note that the sample size calculations were adjusted in December 2016 to accommodate the randomisations in the seamless design from Phase II to Phase III. The significance level was adjusted to 0.008333 to reflect the fact that 6 randomisations were in this position and thus required adjustment of the $p$ value to $0.05 / 6$. The sample size required with a 2 -sided alpha of 0.008333 and power or $80 \%$ for a hazard ratio of 0.69 as analysed by the logrank test is sufficient to detect 350 events across the two arms being compared. Consequently, full recruitment will be 420 patients for the full-scale phase III trial, and this was reflected in the trial protocol from 2017 onwards

\section{Recruitment $\{15\}$}

It was hoped, at the outset of the trial, that approximately 180 patients per annum would be recruited to the Programme, based on recruitment into the predecessor non-intensive arm of the AML16 trial. The trial remains available for many centres and patients in the UK; recruitment has been significantly supported by collaborative groups around the world. Recruitment varied throughout the program based on availability of novel options and the wider treatment landscape. Recruitment to each of the reported arms thus far has taken approximately 1 year, in line with expectations.

\section{Assignment of interventions: allocation}

\section{Sequence generation $\{16 \mathrm{a}\}$}

Patients are, and were, randomized (using an online trial-specific system, hosted by the Sponsor) between all available treatments via a minimization process, which balances treatment arms with regard to sex, age (age groups 60-64, 65-69, 70-74, 75+), disease type (de novo AML, MDS or secondary $\mathrm{AML}$ ) and white cell count at entry. Randomisation balance is monitored and reported to the Trial Management Group throughout the trial. 


\section{Concealment mechanism \{16b\}}

Patients are assigned a four-digit trial identification number by the on-line randomization system, on a consecutive basis, independent of trial centre. Therefore, treatment is concealed from the participating centre and the trial management team until the point of allocation.

\section{Implementation $\{16 \mathrm{c}\}$}

The allocation sequence is generated automatically by the online system, and ongoing minimization is based on the factors outlined above. Patients with AML are enrolled by personnel at participating centres - centres are activated on the online system by the central management team, based on provision of approval documentation and user-specific accounts set up to enable randomisation to be undertaken online.

\section{Assignment of interventions: Blinding}

\section{Who will be blinded $\{17 \mathrm{a}\}$}

Patients and clinical teams at participating centres, and the central trial management team, are all unblinded to treatment allocation. Assessment of clinical outcome is undertaken at participating centres alongside delivery of allocated treatment. Quality of Life data is also gathered and assessed by the central trial management team.

\section{Data collection and management}

\section{Plans for assessment and collection of outcomes $\{18 \mathrm{a}\}$}

Collection of data is via a standard patient record book, provided to all participating centres, and reproduced online. Data is entered online at participating centres, utilizing user-specific accounts generated by the central trial team. Toxicities during treatment are reported by participating centres in line with CTCAEv3.

The central trial team collects, manages, cleans, analyses and reports the trial data in accordance with the SOPs at the Centre for Trials Research (a CRUK and UKCRC-accredited clinical trials unit). Collection of clinical data from centres may be subject to review during a monitoring visit to a participating centres. Patient-reported data, such as Quality of Life questionnaires, are not subject to data cleaning.

\section{Plans to promote participant retention and complete follow-up $\{18 b\}$}

In addition to data collected during the treatment phase, bone marrow and survival status of patients in this trial is updated on an, approximately, annual basis using data gathered from participating centres. Patients may, at the discretion of their treating clinical team, revert to a different standard treatment if their disease does not respond to novel allocated treatment. However, all patients in the trial are followed up until death and are analysed on an intention-totreat basis.

\section{Data management $\{19\}$}

Data cleaning is an ongoing process in accordance with CTR SOPs and a trial-specific data management plan. The protocol outlines the data that is required for collection. Participating centres are able to view data entered for their patients, and are required to ensure accuracy of data. The online data collection tool includes validation that will not allow receipt of impossible data. Data ranges on some key fields prompt automated comments during trial entry to query improbable or unusual data. 
Patient information sheets include specific comment regarding named information - informed consent allows Sponsor to hold named data, with a view to potentially accessing additional data about the patients in this trial from routine datasets. Patient names may be held in the clinical trial database, but are not visible to the trial team and may not be shared with any research collaborators. Data is published or reported by arm and does not allow trial patients to be identified.

\section{Plans for collection, laboratory evaluation and storage of biological specimens for genetic or molecular analysis in this trial/future use $\{33\}$}

A bone marrow or peripheral blood sample is requested for each patient at trial entry. A molecular genetic analysis is performed in the Department of Haematology, Cardiff University, in order to assess the status of the patient. This analysis is particularly important in analysis of particular arms of the trial (for instance, the AC220 drug is potentially more efficacious in FLT3+ve patients) however, the analysis does not direct patient treatment allocation and lack of sample provision does not preclude the patient from entering the trial.

Many patients undergo a bone marrow assessment at a local or district centre and may receive their formal diagnosis at a regional centre some weeks later. In this frail, elderly population it is not considered ethical to mandate that the patient be re-marrowed, so a peripheral blood sample is often provided (where circulating disease is present).

Specific consent is required in order for samples to be placed in the Cardiff University Biobank (CUBB). These samples are pseudonymised and made available to the wider research community for ongoing research - this process is overseen by HTA-compliant processes. While the trial is still ongoing, the processes for sample collection, processing and banking continue.

\section{Statistical methods}

\section{Statistical methods for primary and secondary outcomes $\{20 \mathrm{a}\}$}

Analysis of primary outcomes

The main analyses will be performed using standard contingency table and log-rank methods based on the intention to treat principle, i.e., all patients eligible at the time of randomisation will be included in the analysis, irrespective of protocol compliance.

Time-to-event outcomes will be analysed using the logrank test and reported hazard ratios and confidence intervals derived from the (o-e) and V statistics. Dichotomous outcomes (e.g., CR) will be analysed using the Mantel-Haenszel test, as this produces equivalent statistics to the logrank test and allows stratified analyses to be performed and presented analogously to the survival outcomes. Hematopoietic recovery will be analysed using the logrank test. Patients who do not recover their count will be censored as stated in the definition of the endpoint.

\section{Analysis of secondary outcomes}

Toxicities will be reported as tables (of numbers experiencing grades 0-4) together with average grades. Treatments will be compared using the Wilcoxon rank-sum (Mann-Whitney U) or KruskalWallis test as appropriate. The same test will be used to compare supportive care requirements (i.e. the number of units of blood and platelets given, the number of days spent on IV antibiotics and the number of days spent in hospital). Adverse events will be categorised and tabulated to identify if there is excessive incidence in one or other treatment groups. Responses from quality of life assessment will be presented using descriptive statistics.

For each analysis required a more detailed Statistical Analysis Plan (SAP) is developed by the trial statistician according to the CTR SOPs. This is then approved by a senior statistician and chief investigator. 


\section{Interim analyses $\{\mathbf{2 1 b}\}$}

Interim analyses will be presented in confidence to the Independent Data Monitoring Committee (IDMC). Full details are given in the interim statistical analysis plan. This evaluation will take place in three stages. The precise approach for performing these initial assessments of effectiveness will depend on the anticipated modality of the treatment. For some treatments, it is anticipated that any improvements in survival will be achieved by increasing the number of durable remissions. For others, such as hypomethylating agents, it may be possible to improve survival without increasing the remission rate. Consequently, for each novel agent assessment will follow one of two strategies.

For treatments where the proposed effect is to improve survival by inducing a greater number of remissions, recruitment will proceed until at least 50 patients have entered each comparative arm (LD Ara-C and novel therapy). This component will then be analysed using CR as endpoint. While this assessment is taking place, recruitment may continue subject to the wishes of the IDMC. If the arm appears sufficiently promising, then recruitment will continue until 100 patients are in each arm. At this point, a similar analysis on CR will be undertaken. If, on the basis of examining the data from the first 100 patients in each arm, the novel treatment is sufficiently promising then recruitment will continue to 200 patients per arm as a Phase III trial, when the trial endpoints will be changed to CR, relapse and OS. However, if at either of the earlier analysis points, the judgement is that the treatment is unlikely to hold promise, the comparison will be discontinued. The choice of $\mathrm{CR}$ as endpoint in the initial comparisons is driven by two considerations. First, patients in the non-intensive part of AML14 who failed to enter remission had very poor prognosis. Thus, it is reasonable to assume that any treatment where the aim is to induce remission is unlikely to be able to improve OS without also improving remission rates in this group. $\mathrm{CR}$ is also an endpoint for which data become available very quickly, so a decision on whether there is sufficient evidence of improved CR rates to persist with a given therapy can be made in an expeditious fashion.

\section{Stopping Rules}

The decision on whether to proceed with a comparison will depend on the experimental treatment meeting certain pre-set improvements in CR rate compared to the LD Ara-C arm of the trial. The precise choice of cut-off at the two interim monitoring points (50 and 100 patients per arm) may change dependent on the treatment being tested: for example, for an inexpensive, welltolerated drug one would be likely to accept a smaller improvement than if the drugs were expensive, toxic and difficult to administer. For each treatment, the IDMC will be issued with a detailed monitoring plan, incorporating guidelines for deciding whether to stop or continue with a novel therapy. For the treatments in this protocol other than sapacitabine, the default stopping rule is as follows: assuming a CR rate of $15 \%$ with LD Ara-C and aiming to identify new treatments that produce a CR rate of $30 \%$ or more, a minimum of 50 patients will be accrued to each novel therapy arm; if the improvement in CR rate in the novel therapy arm is less than $2.5 \%$ (15\% to $17.5 \%$ ), that arm will be closed (with a $7 \%$ chance of rejecting a treatment with a true CR rate of $30 \%$ or more); if the difference in CR rates exceeds $2.5 \%$, the arm will continue to 100 patients. At this point, if the improvement in CR rate in the novel therapy arm is less than $7.5 \%$, that arm will be closed (with a further $8 \%$ chance of rejecting a treatment with a true CR rate of at least $30 \%$ ); if the improvement in CR rate exceeds $7.5 \%$, the comparison will continue to the full 200 patientper-arm trial.

While the benefit derived from some treatments is expected to come from an increase in the number of durable remissions, some drugs, notably hypomethylating agents give the promise that survival may be prolonged even without the achievement of CR. In this case, an interim analysis based on CR is inappropriate. Instead, analyses will be based on OS once one quarter and one half of the required number of events have been seen - in this case this equates to 85 and 170 events. 
At this point, the decision to stop or continue will be made on the basis of the hazard ratio for OS. A doubling of survival from $10 \%$ to $20 \%$ at two years equates to a hazard ratio of 0.70 . Thus, for drugs such as sapacitabine, where remission is not viewed as a prerequisite for survival, after 85 events, the hazard ratio (drug versus LD Ara-C) must not exceed 1; and after 170 events it must not exceed 0.85 (i.e. half the required effect).

The formal decision to stop a randomisation will rest with the Trial Steering Committee and Sponsor on advice from the IDMC.

Methods for additional analyses (e.g. subgroup analyses) $\{20 \mathrm{~b}\}$

Final analysis of the primary endpoints will be stratified by the following factors:

- Age (60-64, 65-69, 70-74 and 75+).

- WHO performance status ( $0=$ normal activity, $1=$ restricted activity, $2=$ in bed $<50 \%$ waking hours, $3=$ in bed $>50 \%$ waking hours, $4=$ completely disabled).

- Type of disease (1=de novo, 2=secondary AML/MDS, 3=high risk Myelodysplastic Syndrome).

- White blood count $(<10,10-49.9,50-99.9$ and $100+)$.

- Cytogenetic risk group (normal/abnormal/unknown).

- Sorror score and co-morbidities

Results will be summarised by the stratification variables including formal tests of interaction with treatment and will be presented as a forest plot.

Where appropriate, some sub-group analyses were indicated - for instance, in the AC220 randomisation, the FLT3+ve group were reported as a sub-group due to the type of agent (tyrosine kinase inhibitor).

\section{Methods in analysis to handle protocol non-adherence and any statistical methods} to handle missing data $\{20 \mathrm{c}\}$

Missing values will be examined and described. No multiple imputation is planned for the analysis.

\section{Plans to give access to the full protocol, participant level-data and statistical code} $\{31 c\}$

Access to data is subject to the standard operation procedures of the Sponsor, and requests for data will be reviewed accordingly. Data sharing is actively encouraged by accredited clinical trials units.

\section{Oversight and monitoring}

Composition of the coordinating centre and trial steering committee $\{5 d\}$

Ian Thomas, Senior Trial Manager

Laura Upton, Trial Manager

Cono Ariti, Senior Statistician

Mia Sydenham, Data Manager

Trial Management Group (TMG) members:

Dr Steve Knapper, University Hospital of Wales (Clinical coordinator)

Professor Richard Clark, University of Liverpool (Clinical coordinator)

Chris Shaw, Cardiff University (Sponsor/Research Governance Officer)

Amanda Gilkes, Cardiff University (Tissue Banking)

Trial Steering Committee (TSC) members:

Professor Don Milligan, Chair (retired), University of Birmingham

Roland Walter, Chair (current), Fred Hutchinson Cancer Research Centre

Christoph Schliemann, independent/international clinical oversight, University of Muenster 
Eftychia Psarelli, independent statistician, University of Liverpool

Dr Clare Rowntree, independent clinical oversight, University Hospital of Wales

Composition of the data monitoring committee, its role and reporting structure $\{21 a\}$ Independent Data Monitoring Committee (IDMC) members

Professor Andrew Lister (Chair), Emeritus Professor, Barts Cancer Institute

Professor Graeme MacLennan, University of Aberdeen, independent statistician

Professor Janet Dunn, University of Warwick, independent statistician

Dr Mark Drummond, independent clinician, Beatson Cancer Centre, Glasgow

Dr Gail Jones, independent clinician, Newcastle upon Tyne Hospitals NHS Foundation Trust

Dr Bernard Ramsahoye, independent clinician, University of Edinburgh

The IDMC is comprised of independent members and meets at defined timepoints in order to review emerging data from treatment arms and decide on progression or otherwise of treatment arms. IDMC may decide an arm may proceed to the next analysis, should close or they may decide that further data is required to inform their decision. The IDMC reports to the TSC (see above), solely in regard to matters outlined in the IDMC charter.

\section{Adverse event reporting and harms $\{22\}$}

Toxicities, or AEs, are routinely reported by participating centres as part of the data requirements for the trial, as outlined in the protocol. This data is reported as it emerges and ordinarily is reported at the end of a course of treatment. Toxicities are reported to the IDMC - safety (toxicity profile) forms a critical element of the decision as to whether a novel agent may progress to further analyses. Tabulated toxicity data also forms part of published data about specific treatment arms.

Where toxicities meet the protocol definition for Serious Adverse Event reporting, a report form is submitted to the trial centre within 24 hours of the participating centre becoming aware of it. Additional reporting of SAEs is subject to CTR SOPs relating to SUSARs, DSURs, etc. in line with regulatory requirements.

\section{Frequency and plans for auditing trial conduct $\{23\}$}

General trial conduct will be overseen by the TMG in accordance with the Trial Monitoring Plan, which itself is in accordance with CTR SOPs and accepted monitoring guidelines. Review of trial conduct includes level of protocol non-compliances, deviations, etc., data return, including SAE reporting, and management of IMPs. Conduct of the trial team is monitored by the CTR Quality Management System, administered by the Quality Assurance group.

\section{Plans for communicating important protocol amendments to relevant parties (e.g. trial participants, ethical committees) $\{25\}$}

Amendments to the protocol are defined as either substantial or non-substantial by the Sponsor. Communication of non-substantial amendments are agreed by the TMG.

Processing, submission and communication of substantial amendments is in accordance with regulatory requirements, CTR SOPs and Sponsor requirements. Regulatory requirements in the UK include approval, where appropriate, by Medicines and Healthcare products Regulatory Agency, Research Ethics Committee (Wales REC 3), Health Research Authority and Research \& Development departments at participating centres. Additional requirements are in place for participating centres outside of the UK. 
Collaborating pharma companies, IMP release facilities, central laboratories, national lead investigators and funders are informed of substantial amendments in line with the specific requirements of the amendment, and with contractual obligations between parties. Where appropriate, authorization for amendments is sought from these parties in advance of submission to regulators.

\section{Dissemination plans $\{31 \mathrm{a}\}$}

Results from each treatment arm will be published as a completed, clean dataset becomes available to the trial team. While this process may require relatively long-term follow-up, the progress of treatment arms through the different analyses is reported to investigators in real-time as it impacts on the treatment options available to newly-randomised patients - where a treatment arm does not demonstrate enough promise to pursue, the arm will be closed and investigators will be informed in advance of results being published.

Sponsor expects investigators to inform ongoing patients of trial updates on a discretionary basis.

\section{Discussion}

Challenges in delivering platform trials such as this are subject to ongoing discussions between accredited clinical trial units (CTUs), and may result in future guidance document and publications. Particular challenges include ensuring stable standard of care across the trial, considering the impact of new licensed agents in the disease area, defining the end of trial while patients on several arms may still require follow-up and balancing funding requirements against a changing regulatory landscape. In the LII trial, recruitment has been variable throughout the trial period and changes as different novel agents become available. Describing the current status of the trial at any given time to a patient has presented challenges to some participating centres.

\section{Trial status}

The trial is currently following protocol version 11.1, which was approved in the UK in November 2019. Recruitment to this trial program opened in December 2011 and the first patient was allocated to trial treatment in January 2012. It is expected that recruitment will be completed in Q1 of 2021. 


\author{
Abbreviations \\ AML: Acute myeloid leukaemia \\ $\mathrm{Cl}$ : Chief Investigator \\ CR: Complete remission \\ CRi: complete remission with insufficient count recovery \\ CTR: Centre for Trials Research \\ CTU: Clinical trials unit \\ CUBB: Cardiff University Biobank \\ HR: hazard ratio \\ HTA: Human Tissue Act \\ IDMC: Independent data monitoring committee \\ LD Ara-C: low-dose cytarabine \\ LI1: Less-intensive 1 \\ MDS: myelodysplastic syndrome \\ MHRA: Medicines for Human use Regulatory Agency \\ NCRI: National Cancer Research Institute \\ OS: overall survival \\ REC: Research Ethics Committee \\ RFS: relapse=free survival \\ SAP: statistical analysis plan \\ SOP: Standard operating procedure \\ TMG: Trial management group \\ TSC: Trial steering committee \\ UKCRC: United Kingdom Clinical Research Collaboration \\ WHO: World Health Organisation
}




\section{Declarations}

\section{Acknowledgements}

Professor Richard Adams provided senior CTR oversight and Charlotte Wilhelm-Benartzi provided senior statistical oversight. Mel Varley and Victoria Boyland provided data management support during the trial. Matt Robinson-Burtt provided information services support.

Lars Kjeldsen led the contribution from the leukaemia group in Denmark; Ruth Spearing, Lucy Pemberton and Clare Hemmaway led the contribution from the leukaemia group in New Zealand; Herve Dombret led the contribution from the group in France and Andrew Wei and the ALLG led the contribution from the group in Australia.

\section{Authors' contributions $\{31 \mathrm{~b}\}$}

$\mathrm{CA}, \mathrm{CWB}$ and $\mathrm{RH}$ conducted and supervised the analysis of the trial data. $\mathrm{MD}, \mathrm{AKB}$ and $\mathrm{MC}$ were trial Chief Investigators. RH, AKB, MD, NR and IT were major contributors to the trial design, conception and conduct. LU and IT supervised acquisition of trial data. LK and CH provided substantial supervision of international trial conduct.

\section{Funding $\{4\}$}

The trial was supported by central funding from Blood Cancer UK (formerly Bloodwise, formerly Leukaemia Lymphoma Research). Bloodwise reviewed and approved the design of the trial and subsequent amendments and additions of trial arms, but no part in the collection, analysis or interpretation of the data.

Additional funding from the collaborating pharma companies (Cyclacel, Daiichi Sankyo (formerly Ambit Biosciences), Sunesis Pharmaceuticals, Synta Pharma, Karyopharm, Cell Therapeutics Inc, Celgene and BioCancer Therapeutics Inc) supported delivery of specific trial arms, in particular provision of novel agents to participating centres. These pharma companies did not have any role in collection, analysis or interpretation of data or in the production of this manuscript. The trial Sponsor, Cardiff University, provided legal and research governance support.

\section{Availability of data and materials $\{29\}$}

Datasets generated and/or analysed during this trial are not publicly available as they represent intellectual property on behalf of the Sponsor in collaboration with research partners. However, applications for access to the data, in a pseudonymised format, may be made to the corresponding author and will be reviewed in line with existing CTR SOPs.

It is the intention of the research group and the NCRI AML working group, who supported the trial, to make data available for patient benefit, wherever possible.

\section{Ethics approval and consent to participate $\{24\}$}

Informed consent will be obtained from all study participants. This trial Programme included administering of novel agents to patients, and collection of biological tissue, and therefore required Ethical oversight. The trial Programme was reviewed and Ethically approved by Wales REC (Research Ethics Committee) 3.

\section{Consent for publication $\{32\}$}

Not applicable - data pertaining to individual patients is not included.

\section{Competing interests $\{28\}$}

The authors declare they have no competing interests 
Page 19 of 20 


\section{Author details}

Senior Research Fellow:

Ian Thomas, Centre for Trials Research, Cardiff University

Chief Investigator:

Dr Mike Dennis, Consultant Haematologist, Director of the Haematology and Transplant Unit, The Christie Hospital, Manchester, UK

Co-Cl:

Prof Mhairi Copland Professor of Translational Haematology and Honorary Consultant, Director of the Paul O'Gorman Leukaemia Research Centre and Head of Experimental Haematology, College of Medical, Veterinary and Life Sciences, Institute of Cancer Sciences, University of Glasgow Clinical Coordinators:

Prof Alan Burnett, Blackwaterfoot, Isle of Arran, UK

Prof Nigel Russell, Consultant Haematologist, Guy's Hospital NHS Trust (Emeritus Professor of Haematology,

Nottingham University)

Trial Design and statistical oversight:

Prof Robert Hills, Professor of Medical Statistics, Nuffield Department of Population Health, University of Oxford

Statistical analysis:

Mr Cono Ariti, Research Fellow (Statistics), Centre for Trials Research, Cardiff University

Prof Robert Hills, Professor of Medical Statistics, Nuffield Department of Population Health, University of Oxford

\section{References}

1) Lowenberg B, Downing JR, Burnett AK. Acute myeloid leukemia. N.Engl.J.Med. 1999; 341 (14):1051-62.

2) A. K. Burnett, D. Milligan, A. G. Prentice, A. H. Goldstone, M. F. McMullin, R. K. Hills, and K. Wheatley. A comparison of low-dose cytarabine and hydroxyurea with or without all-trans retinoic acid for acute myeloid leukemia and high-risk myelodysplastic syndrome in patients not considered fit for intensive treatment. Cancer 109 (6):1114-1124, 2007

3) Hills RK, Burnett AK. Applicability of a "Pick a Winner" trial design to acute myeloid leukemia. Blood. 2011 Sep 1;118(9):2389-94.

4) Cheson BD et al. Revised recommendations of the International Working Group for Diagnosis, Standardization of Response Criteria, Treatment Outcomes, and Reporting Standards for Therapeutic Trials in Acute Myeloid Leukemia. J Clin Oncol. 2003 Dec 15; 21(24):4642-9.

5) Quality of Life questionnaires QLQ-c30 https://qol.eortc.org/questionnaire/eortc-qlq-c30/, HADS https://hqlo.biomedcentral.com/articles/10.1186/1477-7525-1-29 and EUROQOL EQ5D https://euroqol.org/eq-5d-instruments/ 